\title{
Computational Psychiatry
}

2.2 Computational Models of Cognitive Control: Past and Current Approaches

Debbie M. Yee and Todd S. Braver 


\section{INTRODUCTION}

A core challenge of cognitive, computational, and systems neuroscience research is to provide a satisfying answer to the following question: how does cognition arise from neural systems? Although researchers have spent decades using variety of tools (e.g., magnetic resonance imaging, electroencephalography, single-unit recordings) to investigate this question, we have only begun to scratch its surface, in terms of understanding how neural substrates work together in synchrony to give rise to complex cognitive processes.

To provide an analogy, imagine listening to a concerto performed by a symphony orchestra. Perhaps you are interested in understanding how the orchestra can blend together so many different sounds from vastly different instruments to give rise to this beautiful masterpiece. In the initial hearing, the piece sounds clearly melodic, lyrical, and filled with multiple complex musical layers that sound cohesive when in concert. However, upon closer examination, it becomes evident that even such complex musical layers can be deconstructed into the contributions from different instruments within the entire ensemble. One approach for understanding the concerto may be to simply to listen one instrument or one section (e.g., attending to a violin solo or the entire violin section when playing the same melody); however, that would only provide a small window into how that specific instrument contributes to the entire piece. Another approach would be to parse out all of the sounds in the piece by instrument, which provides a structural division of the different sounds that comprise the concerto, but neglects the temporal ordering of when the instruments are played, an important aspect of the composition. Perhaps the most insidious problem is that even if we are able to understand the structural and temporal aspects of how each instrument contributes to this specific concerto, the same instruments in this symphony orchestra can also perform a wide variety of other 
compositions (e.g., other concertos, sonatas, ballads) at other periods in time! Thus, the characterization of the violin's contribution in the current concerto may not applicable when considering other musical performances, which makes current analyses efforts not quite as generalizable as we might have hoped.

\section{The Homunculus Problem of Cognitive Control}

The challenge of this problem and the "orchestra concerto" metaphor becomes particularly salient when considering one of the most compelling mysteries of human cognition: how the brain enables human beings to plan, implement, and accomplish the types of controlled, complex, and temporally extended goal-directed behaviors that make-up much of modern daily life (e.g., preparing a multi-course meal, constructing IKEA furniture from an instruction manual, writing a computer program, solving a Sudoku puzzle, figuring out how successfully complete an $\mathrm{MD}$ or $\mathrm{PhD}$ ). In the orchestra metaphor, it would be akin to understanding how the conductor guides the ensemble to put together a beautifully sounding and cohesive concerto performance. This mystery has often been posed as the "homunculus problem," which presents the following conundrum: if control over thoughts and action emerges from brain function, then are there special neurobiological and computational properties that differentiate the components that should be labeled as "controller" from the components that are "controlled"? Does controller/controlled distinction even make sense? And if not, how are we ever going to understand the emergence of intelligent, goal-directed behavior in neurobiological terms?

Within psychology and neuroscience, researchers have often taken a primarily localizationist approach, studying individual brain regions in terms of their associated cognitive functions (Poldrack 2007). At the other extreme, is the integrationist perspective, which focuses 
on the entire brain, parsing it into networks that may be structurally or functionally related (Eliasmith et al. 2012). However, neither of these approaches has yet provided a fully satisfying answer to the fundamental problem of cognitive control. Indeed, as this discussion hopefully makes clear, properly addressing the seemingly intractable homunculus problem likely requires a computational modeling approach. Computational approaches can be utilized in both a reductionist and emergentist manner: deconstructing the mysterious intelligence of the homunculus into hopefully more understandable "dumb" neural subcomponents, while at the same time making clear how complex control functions emerge from the dynamic interactions among these multiple simpler subcomponents of cognitive control.

Computational modeling approaches to cognitive control are uniquely powerful, in that they provide the researcher with a means of generating specific and concrete hypotheses, along with explicit experimental predictions regarding generative and causally efficacious control mechanisms and their influence on brain activity and behavior (Botvinick and Cohen 2014; O’Reilly 2006; O’Reilly, Herd, and Pauli 2010). More broadly, within the cognitive sciences, the utility of modeling approaches has long been established and appreciated (Newell and Simon 1961). Over thirty years ago, David Marr attempted to formalize these approaches by articulating an influential proposal for decomposing and investigating complex cognitive systems across three levels of analysis: the computational, the algorithmic, and the implementational (Marr 1982; Bechtel 1994).

According to the Marr framework, the computational level aims to specify the function that the cognitive system must perform - namely addressing the question "what is the goal of the computation, and why is it functionally relevant to the organism?" In contrast, the algorithmic (or representational) level aims to understand the procedures by which this computation is 
carried out - chiefly focusing on the representation of inputs and outputs, and the algorithm that underlies this transformation. Finally, the implementational level aims to identify the physical mechanisms that carry out this process - specifically, how the brain performs these cognitive functions. These levels of analyses were initially introduced to tackle computational questions in vision, and have been criticized by various researchers as potentially being too rigid to be universally applicable (Dayan 2001). Yet we feel that the Marr framework can be fruitfully applied when considering complementary questions about the neural and computational mechanisms that underlie more complex temporally extended goal-directed behavior. Consequently, we will make use of the framework in model descriptions presented in this chapter, in order to provide a general intuition for how various computational models attempt to address specific questions about cognitive control function.

-- Figure 1 --

\section{Why Cognitive Control?}

The current chapter highlights past and current computational models of cognitive control, and the purpose is two-fold. First, cognitive control is a well-known psychological construct, with a long history of researchers using computational modeling approaches to attempt to explain its underlying cognitive mechanisms (Newell and Simon 1972; Rumelhart et al. 1986; Cohen, Dunbar, and McClelland 1990; Braver and Cohen 2000; Anderson et al. 2008). Second, cognitive control ability is disrupted across a wide range of mental disorders, with a vast body of literature now supporting the hypothesis that cognitive control impairments are prominent in such disorders as schizophrenia, depression, obsessive-compulsive disorder, ADHD, addiction, Alzheimer's Disease and Parkinson's Disease (Lesh et al. 2011; Fales et al. 2008; Halari et al. 2009; Greisberg and McKay 2003; van Meel et al. 2007; Vaidya et al. 2005; Belleville, 
Chertkow, and Gauthier 2007; R. G. Brown and Marsden 1990; Wylie et al. 2010; H. R. Snyder, Miyake, and Hankin 2015). Indeed, it may not be an exaggeration to argue that an impairment of cognitive control, in one form or another, is the core feature of mental illness more generally. Thus, understanding the mechanisms that underlie cognitive control function can provide a crucial window into psychopathology.

Cognitive control is operationalized as the ability to perform task-relevant processing in the face of distractions or in the absence of environmental support, specifically by active maintenance and flexible updating of task representations over time, in order to pursue taskrelevant objectives and behavioral goals (Engle and Kane 2004; Braver 2012; O’Reilly, Braver, and Cohen 1999). A core tenet of cognitive control is the distinction between controlled and automatic processing (Posner and Snyder 1975; Shiffrin and Schneider 1977; Norman and Shallice 1986), which emphasizes the fundamental tradeoff between recruiting and directing cognitive resources to deliberately perform a demanding task versus carrying out less effortful and habitual responses that may require fewer attentional resources, but which also may be less flexible. Typically, the allocation of control depends on the circumstances for demand. In other words, the control of behavior arises from the cognitive demands required to successfully perform a task, and effort allocation arises from the dynamic recruitment of available cognitive processes that can appropriately meet these demands during task performance (Botvinick and Cohen 2014). Some have proposed various computational models and frameworks to understand this tradeoff between effort and automaticity in controlled behavior (Cohen, Dunbar, and McClelland 1990; Schneider and Chein 2003), whereas others have hypothesized that humans perform a cost-benefit analyses between expected payoff and cognitive effort to determine the optimal allocation of cognitive control (Shenhav et al. 2017; Dixon and Christoff 2012). All in 
all, there still remain many unanswered questions regarding the computational and neural mechanisms that underlie cognitive control; which we argue can be more adequately addressed with computational modeling approaches.

As a brief aside, we wish to acknowledge that such computational modeling approaches have been prevalent and successful in advancing understanding for other related, but potentially more specialized cognitive processes, such as attention (Gershman, Cohen, and Niv 2010), learning (Tenenbaum, Griffiths, and Kemp 2006), semantic knowledge (Rogers and McClelland 2004), and memory (Polyn, Norman, and Kahana 2009). Thus, while this chapter will focus primarily on cognitive control, we hope that the reader may extrapolate these principles to obtain a broader perspective for how computational models can be used to study other cognitive systems.

\section{Roadmap to this Chapter}

This chapter contains two main sections. First, we will provide a brief review of several key computational models that have been influential in advancing understanding of cognitive control mechanisms. This review of such models are not meant to be comprehensive, but will hopefully provide a useful primer for readers to become familiar with classical and current computational models of cognitive control, with the understanding that the principles behind these models can also be extended to other related models. Next, we discuss key features of computational models that make them particularly useful and generative in guiding further research efforts (i.e., what "tests" can we run to determine whether a computational model can make accurate and generalized predictions about controlled behavior?). The chapter concludes with a concrete example of how such modeling frameworks can be used to make predictions in 
mental illness, with some speculation about how cognitive control function breaks down in schizophrenia, a psychiatric disorder hypothesized to be associated with cognitive control impairment.

\section{PAST AND CURRENT MODELS OF COGNITIVE CONTROL}

A broad range of computational models have played a prominent role in the development and understanding of cognitive control theory and its underlying mechanisms, including those that have primarily arisen from symbolic modeling traditions, such as those involving production system architectures (ACT-R, Anderson 1996; EPIC, Kieras and Meyer 1997). At the other end of the spectrum are models arising from the computational neuroscience tradition (Wang 2013), some of which are covered in other chapters. To narrow the scope of this particular brief chapter, we focus on four contemporary models that address challenging and unique computational problems integral to cognitive control function, and which have also played an influential role in advancing research within this domain:

- Working Memory and Gating Mechanisms in Basal Ganglia-Prefrontal Cortex Interactions

- Prediction and Outcomes in Anterior Cingulate Cortex

- Hierarchical Organization of Prefrontal Cortex

- Interactions of Cognitive Control with Reinforcement Learning and Decision Making

\section{Working Memory and Gating Mechanisms in Basal Ganglia-Prefrontal Cortex Interactions}

One of the earliest models of cognitive control focused on the following computational question: how does the brain determine what information is relevant to be maintained (i.e., in 
working memory) during the pursuit of task goals, and when should this information be updated with newer task-relevant information? A potentially useful analogy for visualizing this issue is the concept of 'mental blackboard,' which describes the dilemma of deciding between when learned information in working memory should be kept, or instead erased and overwritten (Baddeley 1986). Early neurocomputational models attempted to use attractor models to understand the mechanisms that underlie robust active maintenance of working memory against irrelevant distractors (Changeux and Dehaene 1989; Zipser et al. 1993; Cohen, Braver, and O’Reilly 1996; Compte et al. 2000; Durstewitz, Seamans, and Sejnowski 2000; Deco and Rolls 2003). However, a major limitation of these models is their lack of a mechanism for precisely updating working memory when newer, task-relevant information is introduced. This tension between these two working memory functions is difficult to reconcile, as they inherently contradict each other - active maintenance increases resistance to distractors, whereas flexible updating makes the system more vulnerable to distraction. Thus, the computational challenge lies in building a model which can explain how a system regulates the fundamental trade-off between learning when to actively maintain context representations (i.e., task-relevant information that is internally represented) to achieve controlled processing versus rapidly updating new information into working memory, a core problem of cognitive control (O'Reilly, Braver, and Cohen 1999; Braver and Cohen 2000).

One approach towards understanding the computational mechanisms that underlie this trade-off in cognitive control function comes from the "parallel-distributed-processing" approach (also dubbed "connectionist" or "neural network" models in the literature). This approach integrates basic computational principles of information processing with hypotheses about the types of functional specializations that putatively occur within different brain systems, thus 
enabling biologically-based computational models of higher cognitive processes, such as attention, memory and language (Rumelhart, McClelland, and PDP Research Group 1987; Cohen, Dunbar, and McClelland 1990). A key aspect of the connectionist approach when applied to the domain of cognitive control is that these models view control as arising from the interaction of multiple relatively simple elements (e.g., neurons or neural assemblies that perform local processes within a single brain system or unit). Thus, the models emphasize how cognitive control functions emerge from a network of brain regions activated interactively and in parallel, rather than the more historical modular approach of localizing cognitive function to a single brain region (Hinton 1984; O’Reilly 2006).

A recent well-established model for cognitive control function from within the connectionist tradition is the prefrontal cortex and basal ganglia (PBWM) model developed by Frank, O'Reilly, and their colleagues (Frank, Loughry, and O'Reilly 2001; O'Reilly and Frank 2006; Hazy, Frank, and O'Reilly 2007). In the PBWM model, the prefrontal cortex (PFC) and basal ganglia (BG) interact to solve the maintenance versus updating problem posed at the beginning of this section, by implementing a flexible working memory system with an adaptive gating mechanism. This proposed selective gating mechanism is an elegant algorithmic solution for resolving this computational question, as it provides two separate modes of working memory that optimize active maintenance and flexible updating, respectively (Figure 2a). Specifically, working memory is insulated from distractor signals (i.e., irrelevant sensory input) when the gating mechanism is closed, but is receptive to utilizing information from such sensory signals when gating mechanisms are open. However, the introduction of this gating mechanism then begs the following question: how does the brain know when to open or close the gate? In other words, who or what controls the gate? 
At the biological (i.e., implementational) level, the PBWM model proposes the PFC facilitates the active maintenance mechanisms for sustaining task-relevant information, whereas the BG provides the selective gating mechanism, which independently switches between updating versus maintenance of information in PFC. Specifically, the key component of PBWM is that the BG performs this selective dynamic gating via disinhibition, and moreover, that this dynamic gating functionality depends upon the dopaminergic system (Figure 2b). In this framework, dopaminergic "Go" neurons in dorsal striatum fire to disinhibit PFC to enable updating of working memory representations in PFC, while "NoGo" neurons counteract this effect to support robust maintenance of PFC working memory representations and resistance to distractions.

-- Figure 2 --

Notably, other computational models have proposed similar gating mechanisms that regulate flexible updating and maintenance of task-relevant representations during working memory, but driven primarily by direct DA projections to PFC (Braver and Cohen 1999; Braver and Cohen 2000). However, a criticism of the global DA firing hypothesis is that this mechanism would not fully explain more complex cognitive tasks in which individuals would need to maintain and update different task representations simultaneously (e.g., remembering to press a button for a specific stimulus only during on context A, but not context B).

Taken together, the PBWM leverages the gating mechanism as an algorithmic solution to the computational problem of switching between active maintenance and flexible updating within working memory mechanisms. This model suggests that the PFC implements active maintenance of task-relevant information, whereas the BG contains selective gating mechanisms which switch between "robust maintenance" and "selective updating" of information held in PFC 
during working memory. Midbrain DA release is hypothesized to modulate this gating mechanism. However, exactly how, when, and where DA firing drives these working memory functions (e.g., only in the BG or also directly in PFC), is a question that remains to be fully explored.

\section{Prediction and Outcomes in Anterior Cingulate Cortex}

Another core computational challenge within the domain of cognitive control is the following: how is the current demand for control evaluated, and in what form is this evaluative signal transmitted? In other words, how is it determined which situations or task conditions require more mental resources (than are currently available) to successfully pursue task goals, and what is the necessary relevant information that underlies this neural computation? This question is difficult to address from a purely theoretical perspective, as 'cognitive demand' is an elusive construct that appears to arise under a wide variety of mentally challenging tasks. Thus, a prerequisite for building a computational solution is first understanding under which experimental conditions demand and elicit greater cognitive control, and additionally identifying relevant behavioral measures as empirical evidence for increased mental or cognitive effort in cognitively demanding tasks.

A plethora of work has identified a wide range of tasks with behavioral measures that demonstrate selective recruitment of cognitive control (e.g., Stroop, N-back, stop signal, Erikson flanker) (Botvinick, Cohen, and Carter 2004; Ridderinkhof et al. 2004; Braver and Ruge 2006). For example, in the Stroop task, one must override the prepotent response to read a word, in order to perform the correct task of reading the color ink of the word. In the N-back, one must respond if the stimulus that is currently presented matches a stimulus presented $\mathrm{N}$ trials back 
(e.g., in a 2-back task, the subject must respond if current stimulus matches the one presented 2 slides ago). In the stop-signal (or change signal) task, one must remember whether to maintain or cancel a behavioral response depending on whether or not a stop signal (or change cue) is presented. In the Erikson flanker task, a centrally presented stimulus indicates the relevant behavioral response, and distractor cues placed on both sides of the central stimulus are either be congruent or incongruent with the central cue, with the latter condition requiring greater cognitive control to perform the task accurately. Critically, all of these tasks contain experimental conditions that reliably increase cognitive control demands in a transient, trial-bytrial manner (i.e., the cognitive system monitors ongoing responses and adjusts to the level of cognitive control needed on the current trial). Likewise, they include specific behavioral measures that reflect this enhanced cognitive control demand (e.g., Stroop interference effect, stop-signal reaction time).

A well-established finding is that canonical control tasks, such as the ones listed above, consistently co-activate the dorsolateral prefrontal cortex (dlPFC) and the dorsomedial PFC (Egner 2009; Duverne and Koechlin 2017), specifically a region that spans the dorsal anterior cingulate cortex (ACC) and pre-supplementary motor area (pre-SMA) (Duncan and Owen 2000; Duncan 2010). The dlPFC is thought to play a primary role in actively maintaining representations of task goals and the associated actions (or behavioral rules) needed to achieve them, whereas the ACC is involved in signaling when more control should be implemented by the dlPFC to accomplish task goals. It is generally accepted that the interaction between these two brain regions is important for dynamically adjusting cognitive control. Many have argued for the role the ACC as a locus of cognitive control (Holroyd et al. 2004; Kerns 2004), although 
there remains much controversy over what actual information is represented by the ACC and signaled to the dIPFC to indicate that cognitive control is needed during tasks.

Several prominent theories regarding the interpretation of the ACC's computational role in cognitive control have arisen in recent years, including the detection of error signals (Gehring et al. 1993; Holroyd et al. 2005), reinforcement learning (Holroyd and Coles 2002), conflict monitoring (Botvinick et al. 2001; Botvinick, Cohen, and Carter 2004), error likelihood (Carter et al. 1998; J. W. Brown and Braver 2005), cost-benefit analyses of implementing control (Shenhav, Botvinick, and Cohen 2013), and even uncertainty in the environment (Behrens et al. 2007). Interestingly, although there lacks a consensus between these differing accounts of ACC function, all of them refer to processing of signals that give rise to enhanced cognitive control, which then begs the need for a unifying framework.

To reconcile these divergent perspectives, the prediction response-outcome (PRO) model proposed a unifying algorithmic account of ACC function in discerning when to recruit cognitive control (Alexander and Brown 2011; Alexander and Brown 2014). The PRO model contains two parts. First, the model learns to predict multiple likely outcomes of various chosen actions, regardless of whether these outcomes or good or bad (i.e., response-outcome learning). Second, this model detects discrepancies between actual and predicted outcomes and uses this prediction error signal (i.e., actual outcomes - expected outcomes) to update and refine subsequent predictions. Moreover, a key aspect of this prediction error signal is that it also signals "negative surprise", when an expected outcome does not occur. This form of negative surprise signal can indicate not only when an unexpected error occurs, but also when the response is slower or more equivocal than expected (which is likely to happen on trials associated with high response conflict) 
-- Figure 3 --

At the implementational level, the PRO postulates that separate neural signals within ACC represent outcome prediction and prediction error (negative surprise). Specifically, the model suggests that the prediction signal reliably increases immediately prior to when the mostly likely outcome will occur, whereas the negative surprise signal will reliably activate after the action that produces an unpredicted outcome has occurred. Critically, these hypotheses have been tested empirically across multiple tasks (e.g., change signal task, Erikson-flanker), as well as across different types of neural data (e.g., fMRI BOLD activity, ERP, monkey single unit neurophysiology). Such empirical validation of the PRO demonstrates the utility this model as a useful generalizable computational framework for the underlying calculations for how ACC signals increased need for cognitive control. Recent efforts have attempted to expand this account to include hierarchical representation within ACC and dlPFC (Alexander and Brown 2015), a topic relevant to the next section. Other recent efforts have attempted to link ACC signals with more affective/motivational quantities (Vassena, Holroyd, and Alexander 2017); these include the Expected Value of Control (EVC; Shenhav, Botvinick, and Cohen 2013) and related accounts (Holroyd and McClure 2015; Westbrook and Braver 2016), that postulate that ACC regulates the allocation and persistence of cognitive effort based on signals indicating the current subjective motivational (and/or hedonic) value of task and goal outcomes.

\section{Hierarchical Organization in Prefrontal Cortex}

A third computational question of control relates to the issue of abstraction. How can a 'high-level' goal constrain and implement a 'lower-level' goal? As an example, imagine the following scenario: you hear the phone ring, and you have an instinctive impulse to lift it up 
from the receiver to answer it. However, context plays an important role in your action plan, so while you might automatically answer the phone in your own home, you would inhibit this tendency to answer a ringing phone at your friend's home. Yet, you might switch your action plan if your preoccupied friend asks you to answer the ringing phone on their behalf. This example articulates a fundamental computational challenge of implementing task goals specifically, how do humans utilize contextual representations and higher-level goals to guide action selection during pursuit of lower-level goals, and how does the brain implement this type of hierarchical control?

One promising algorithmic solution for this perplexing question is the concept of hierarchical organization of task goal representations. The notion of applying hierarchical structure to parse complex systems into subordinate and interrelated subsystems has long been established, with subsystems being further subdivided into 'elementary' units (Simon 1962). Similarly, some have argued that control signals used to guide behavioral actions, based on internal plans and goals, can also be subdivided into sensorimotor, contextual, and episodic control (Koechlin, Ody, and Kouneiher 2003; Koechlin and Summerfield 2007). Critically, this information-theoretic model (i.e., based on principles from information theory; Shannon 1948), which has also been termed the "cascade model", postulates that division of control is based on the temporal aspect of when control is implemented. In other words, actions selected based on temporally proximal stimulus would be lower on the hierarchy, whereas actions selected based on past information that is actively maintained in conjunction with the recent stimulus would be higher on the hierarchy. According to this framework, greater demand for cognitive control can also be formalized as the amount of information actively maintained over longer time periods for behavioral action selection. As a brief aside, it is worth noting that others have also utilized 
hierarchical frameworks to understand temporal abstraction in behavior (Cooper and Shallice 2006), but the primary thrust of this work has been to use reinforcement learning to subdivide temporally abstract complex action plans (i.e., 'options') into simpler behaviors, an adaptive and efficient encoding strategy relevant for understanding structured abstract action representations (Botvinick 2008; Botvinick, Niv, and Barto 2009; Solway et al. 2014; Holroyd and Yeung 2011). -- Figure 4 --

At the neural level, the cascade model implements hierarchical cognitive control along the anterior-posterior (i.e., rostral-caudal) axis of lateral PFC, with control signals higher up in the hierarchy represented in more anterior cortical regions (Koechlin, Ody, and Kouneiher 2003; Badre 2008; Badre and D'Esposito 2009). Although it is well accepted that PFC subserves highlevel cognitive function and cognitive control, researchers have only recently attempted to build a parcellation scheme of this large brain region according to a functional organizing principle (Fuster 2001). Evidence from human neuroimaging studies supports the hypothesis of hierarchical representation, with more anterior regions of lateral PFC being activated when cognitive control is implemented for past information, and posterior regions being activated during action selection from more immediate information (Velanova et al. 2003; Braver and Bongiolatti 2002; Braver, Reynolds, and Donaldson 2003; Badre and D'Esposito 2007; Nee and Brown 2013). Additionally, single-unit studies in non-human primates reveal that primate PFC also appears to be functionally organized according to the rostral-caudal axis, whereby more caudal regions are more involved in direct sensorimotor mappings and more rostral regions involved in higher order control processes that regulate action selection among multiple competing responses and stimuli (Petrides 2005; Shima et al. 2007). Thus, the hierarchical organization of PFC appears to be central to performing the neural computations underlying task 
goal abstraction and action selection, though active research efforts focus on understanding how these divisions in the hierarchy are initially learned (Reynolds and O'Reilly 2009; Frank and Badre 2012), and whether the hierarchical structure is primarily anatomic or dynamic (Reynolds et al. 2012; Nee and D'Esposito 2016).

\section{Interactions of Cognitive Control with Reinforcement Learning and Decision Making}

The fourth and final computational question in this chapter relates to the interaction of cognitive control and learning. In daily life, humans are faced with the challenge of learning a set of actions, sometimes simple or complex, in order to complete a specific task (i.e., a task-set). A related challenge is discerning between knowing when these task-set rules learned in one context can be applied to a novel context, or instead when a new task-set needs to be constructed. For example, in the process of searching for the restroom at a shopping mall, you learn to identify signs that contain the text "Bathroom" with arrows that point to its location. However, while this task-set rule may be pertinent when navigating malls in the United States, this action plan may not be effective when searching for a restroom at a Canadian mall, since the signs may read "W.C." instead of "Bathroom." Broadly speaking, creating a set of behavioral tools not tied to the context in which they were learned is useful, as this strategy enables flexible and efficient learning of task-set rules that can be generalized to novel contexts. However, it is less clear what are the neural computations that underlie how cognitive control is deployed to learn task-sets. Thus, the main motivating computational question is the following: in a new context requiring representation of tasks and task-set rules, is it more effective and efficient to generalize from an existing task-set representation (presumably stably encoded in long-term memory), or to instead build a new representation that is more optimized for the current context. 
In the last decade, many accounts of cognitive control looked to algorithms and approaches from the reinforcement learning literature for inspiration in how task-set and goal representations might be acquired (Botvinick, Niv, and Barto 2009; Dayan 2012). A recent model that directly targeted this learning question is the context-task-set (C-TS) model, which aims to approximate how humans create, build, and cluster task-set structures (Collins and Frank 2013). In this model, the algorithmic solution to the computational question harnesses the power of both reinforcement learning and Bayesian generative processes that can infer the presence of latent states. Specifically, the model is designed to accomplish three goals: 1) create representations of task-sets and their parameters, 2) infer at each trial or time point which taskset is relevant in order to guide action selection, and 3) discover hidden task-set rules not already in its repertoire. A key element that drives the learning process is context; here defined as a higher-order factor associated with a lower-level stimulus, which influences which action/motor plan would be selected. The mathematical formulation of the learning algorithm, estimates that when exposed to a novel context, the likelihood of selecting a existing task-set is based on the popularity of that task-set, i.e., that it is appropriate across multiple other contexts. Conversely, the probability of creating a new task-set is inversely proportional to some clustering parameter alpha $(\alpha)$; this parameter serves as a prior that indicates conservativeness, the baseline likelihood that the stimulus-action relationship is thought to be governed by a new rule (rather than an existing one). Further, if a new task-set is created, the model must learn predicted reward outcomes following action selection in response to the current stimulus, as well as determine if the task-set is valid for the given context. If a selected action leads to a rewarding outcome, the model then updates the parameters to strengthen the association between a context and a specific task-set. Thus, C-TS provides a computationally tractable algorithm for task-set learning and 
clustering that not only feasibly links multiple contexts to the same task-set, but also discerns when to build a new task-set to accommodate a novel context. This process has been since dubbed 'structure learning.'

This structure learning process also has an implementational solution, simulated in a biologically plausible neural network model (in the same PDP tradition as the PBWM model), which provides a specific hypothesis about how structure learning occurs in the brain. In particular, the model formalizes how higher and lower level task-set structures and stimulusaction relationships are learned analogously within a distributed brain network involving interactions between PFC and BG. The key functional components of the model are two corticostriatal circuits arranged hierarchically with independent gating mechanisms. The higherorder loop involves anterior regions of PFC and striatum, which learn to gate an abstract task-set and cluster contexts associated with the same task set. The lower-order loop between posterior PFC and striatum also projects to the subthalamic nucleus, which provides the capability of gating motor responses based on the selected task-set and perceptual stimulus. Thus, the execution of viable motor responses are constrained by task-set selection, and conflict that occurs at the level of task-set selection delays the motor response, thus preventing premature action selection until a valid task-set is verified.

Both the algorithmic C-TS and the neural network model lead to similar predictions in human behavior. The demonstration of convergence between these modeling approaches makes clear their joint utility as explanatory tools for understanding the processes that underlie structure learning. Specifically, together these models make an important claim: that humans have a bias towards structure learning, even when it is costly, because such learning enables longer-term benefits in generalization and overall flexibility in novel situations (Collins 2017). 
From a broader perspective, a unique strength of using multiple computational modeling approaches is the ability to provide complementary insight into the cognitive and neural processes that result from the interaction of cognitive control and learning functions. These two models provide an admirable exemplar for integrating computational, algorithmic, and implemental analysis levels, which together formalize a theoretical account that can approximate human implementation of cognitive control processing and structure learning. Thus, while these models specifically target understanding mechanisms of cognitive control, the multi-level approach adopted provides excellent scaffolding for future computational investigation in other cognitive research domains.

\section{DISCUSSION}

Next, we address two relevant issues in evaluating computational models of cognitive control: 1) what are good metrics for determining whether a model provides a useful contribution to our understanding of cognitive control mechanisms?; and 2) how can models in this domain be successfully applied to understand the nature of cognitive control deficits in psychiatric disorders?

\section{Model Evaluation: Determining Whether A Computational Model is Useful}

A famous adage by the British statistician George E. P. Box states the following - "all models are bad, some models are useful." It is generally accepted that most computational models are limited in their ability to account for all observed behavior, and at best typically encompass most of the data variability within a certain limited cognitive domain (e.g., cognitive 
control phenomena related to standard experimental response conflict tasks), but do not generalize well beyond this limited domain, such as to novel tasks or contexts. Another common critique of algorithmic approaches in particular, is that these computations may not necessarily accurately reflect how cognitive processes are implemented on the biological level. For example, while a model may provide a sufficient hypothesis of cognitive control function and account for all the behavioral variance in a task, it is possible that the brain-behavior relationship may arise from a completely different computational or neural process altogether in the brain. Thus, an important step in this approach is model evaluation, i.e., deciding whether a model has utility. In other words, what makes a model useful for advancing cognitive research? Here we describe two complementary metrics for determining the utility of computational models - specifically, examining whether they are descriptive or predictive.

A computational model is descriptive if it provides a detailed explanation that accounts for significant variability of observed data (i.e., how well the model fits the data). Since models provide hypotheses about the data generating process, a descriptive computational model should provide insight into the mechanisms that give rise to the observed behavioral or neural responses in a given task. For example, an indisputable strength of Alexander and Brown's $(2011,2014)$ PRO model is its ability to account for a diverse range of empirical results, related to evaluation of demands for cognitive control, that span across both human and primate studies. Since the PRO model successfully models diverse neural and behavioral data from multiple cognitive control studies, it consequently provides compelling evidence for the hypothesis that predictive neural computation relating actions to outcomes implemented in the ACC and associated medial frontal regions may be a useful signal linked to the engagement of cognitive control. However, although the PRO model formalizes one potential algorithmic explanation for the generative 
process underlying extant data, it may neither reflect the actual neural computations that occur in the brain, nor necessarily accurately predict data outcomes in future studies. Thus, a limitation of this evaluation metric is that while a model with high explanatory power may explain prior data, the proposed mechanism may not be able to explain new data.

Conversely, a computational model is predictive if it describes a generative process that accurately forecasts and extrapolates to novel tasks or contexts. A predictive model contains a specific hypothesis about the neural computations that generate relevant data from one task or context, and incorporates theory to reliably estimate behavioral and neural outcomes in a novel task/context. Collins and Frank's (2013) convergent C-TS and neural network models provide excellent examples of predictive modeling, as both models make accurate predictions of behavioral outcomes in novel tasks/contexts. Critically, a theoretical assumption guiding development of these models is that humans spontaneously build task-set structure in learning problems. This structure learning assumption was tested in empirical studies, validating that the model could generalize to task contexts not previously learned. An important distinction to note is that while both 'descriptive' and 'predictive' computational models describe process mechanisms for how data are generated, the former describes how well the model may fit extant data, whereas the latter describes how well the model generalizes to unseen data.

More broadly and generally, a computational model can serve a very useful function if it is explicitly specified to the degree that it provides a focal point to drive and rejuvenate new research efforts. For example, while there is much controversy over ACC function, computational models have helped to elucidate potentially relevant cognitive mechanisms by providing specific testable hypotheses for empirical study (Botvinick and Cohen 2014; Vassena, Holroyd, and Alexander 2017). Moreover, although models may not always be accurate, they 
can highlight limitations of existing theory (e.g., what can or can't the model predict?), and provide insight into how the theory should be revised in future iterations. The computational models described in this chapter are theory-driven approaches that attempt to describe how the brain implements cognitive control in an explicit way, in contrast to more vague descriptions by conceptual or verbal models. Thus, by attempting to spell out the exact mechanism for how cognitive control systems can be realized, the models described here provide explicit answers to the mysterious 'homunculus' problem of cognitive control. Furthermore, our hope is that such models will eventually be directly useful for elucidating how and why abnormal psychological and neurological processes arise in mental illness.

\section{Cognitive Control Impairments in Schizophrenia}

As an example of the point made above, we conclude this chapter with an example in which computational models of cognitive control have already been directly applied to a psychiatric disorder: specifically to investigate the etiology of cognitive impairments in schizophrenia. A large literature on cognitive function in schizophrenia has reliably established that patients with this illness demonstrate impairments in attention, working memory, episodic memory, and executive functions (Snitz, MacDonald, and Carter 2006). More specifically, an influential hypothesis is that schizophrenia is characterized by disrupted cognitive control, specifically a disturbance in the ability to internally represent and maintain contextual or task goal information in the service of exerting control over one's actions or thoughts (Cohen and Servan-Schreiber 1992; Barch and Ceaser 2012; Lesh et al. 2011; Barch, Culbreth, and Sheffield, in press). A key feature of the account is that such disruptions in cognitive control and context representation are directly linked to dysfunction of the DA neuromodulation in PFC, which has 
long been suggested to be a primary mechanism of pathophysiology in schizophrenia (Meltzer and Stahl 1976; S. H. Snyder 1976; Seeman 1987; Toda and Abi-Dargham 2007; Rolls et al. 2008). In particular, a common view is that at least some of cognitive impairments observed in schizophrenia putatively are related to reduced dysfunctional DA signaling in striatum and PFC, as well as increased 'noise' potentially resulting from increased tonic DA activity or aberrant phasic DA activity (Braver, Barch, and Cohen 1999; Rolls and Grabenhorst 2008; Maia and Frank 2017).

As a direct this hypothesis of dysregulated cognitive control and its relationship to DA and PFC, Braver and colleagues modified an extant computational model of prefrontal cortex function and context processing. Specifically, the goal was to make explicit predictions about behavioral and brain activity patterns that would be observed in schizophrenia patients performing the AX-CPT, a experimental task paradigm designed to distill key aspects of cognitive control and context / goal maintenance (Braver and Cohen 1999; Braver, Barch, and Cohen 1999; Braver, Cohen, and Barch 2002). A key feature of this connectionist computational model, similar to the PBWM model discussed earlier by Frank and colleagues, is that contextual / goal representations are actively maintained in dorsolateral PFC, via mechanisms of recurrent connectivity and lateral inhibition. Most importantly, in this model, DA serves a joint neuromodulatory function within $\mathrm{PFC}$, both gating representations into active maintenance (via phasic signals) and also regulating the persistence of maintenance (via tonic signals) (Braver, Barch, and Cohen 1999; Cohen, Braver, and Brown 2002). Model simulations with this DA neuromodulatory mechanism in PFC bolstered this hypothesis, providing evidence that contextdependent task performance, a key deficit in schizophrenia, is impaired with a noisy DA system (For more specific details, see Braver, Barch, and Cohen 1999). In particular, the model 
predicted very particular patterns of behavioral deficit in the AX-CPT task in participants with schizophrenia, as well disruptions in the temporal dynamics of dorsolateral PFC activity, which were later confirmed experimentally (Barch et al. 2001; Braver, Cohen, and Barch 2002). Nevertheless, it has been difficult to demonstrate direct evidence that such deficits are specifically linked to DA neuromodulatory mechanisms, though recent advances in fMRI techniques have allowed researchers to more precisely measure of dopaminergic phasic signals within the brainstem (D’Ardenne et al. 2012).

Evidence for a related account of contextual / goal representation deficits in schizophrenia was shown in recent work by Chambon and colleagues (Chambon et al. 2008). Here, the goal was to test Koechlin's (2003) cascade model of hierarchical cognitive control in PFC, to see whether it could account for particular patterns of behavioral impairment in individuals with schizophrenia. Interestingly, they observed that sensory and episodic dimensions of cognitive control were preserved in schizophrenic patients, whereas contextual control was impaired compared to matched healthy controls. In the study, patients generated significantly greater errors in tasks that required the ability to maintain context representations, and these impairments were highly correlated with disorganization score (e.g., a measure of disordered thought and behavior). Thus, the evidence is so far consistent with the hypothesis that in schizophrenia the ability to represent and actively maintain contextual or task goal information is disrupted. In future investigations it will be important to more directly test the claims of the cascade model that these deficits map appropriately along the rostro-caudal axis of PFC among individuals with schizophrenia. 


\section{CHAPTER SUMMARY}

This chapter highlighted several computational models that have played a seminal role in guiding theoretical accounts of cognitive control. Critically, we have selected these models because they provide promising testable hypotheses that have already stimulated a great deal of current experimental research, and which are likely to guide future investigations seeking to further elucidate the core neurocomputational mechanisms of that underlie cognitive control. Furthermore, we hope that these models can be a useful primer for understanding computational approaches to cognitive processes more broadly, and how these processes may be disrupted in mental illness. Although computational modeling approaches have played a central role in understanding normative cognitive function (e.g., memory, attention), many of these models have not yet been explicitly tested in psychiatric populations. Thus, we argue that developing accurate mechanistic models of normative cognitive functions can, in principle and in practice, facilitate greater insight into the etiology of psychopathology.

\section{FURTHER READINGS}

These textbooks provide an in-depth introduction into 'parallel distributed processing' (also termed 'connectionist' or neural network') computational models, with the second book a more recent update incorporating more biologically-realistic algorithms and architectures, and explicitly accounting for extant cognitive neuroscience data.

Rumelhart DE, McClelland JL, PDP Research Group. Parallel Distributed Processing, Vol 1. Cambridge, Massachusetts: MIT Press; 1987.

O’Reilly RC, Munakata Y. Computational Explorations in Cognitive Neuroscience. Cambridge, Massachusetts: MIT Press; 2000.

These two articles provide a review of the main scientific questions of cognitive control, and computational approaches that have been proposed to address these questions.

O'Reilly RC, Herd SA, Pauli WM. Computational models of cognitive control. Curr Opin Neurobiol 2010;20:367-77. doi:10.1016/j.conb.2010.01.008. 
Botvinick MM, Cohen JD. The Computational and Neural Basis of Cognitive Control: Charted Territory and New Frontiers. Cogn Sci 2014;38:1249-85.

doi:10.1111/cogs.12126.

An example of how different modeling levels can be utilized to provide converging evidence for cognitive control mechanisms.

Collins AGE, Frank MJ. Cognitive control over learning: Creating, clustering, and generalizing task-set structure. Psychol Rev 2013;120:190-229. doi:10.1037/a0030852.

An example of how a computational model of cognitive control can be applied to make predictions about psychiatric disorder, specifically schizophrenia.

Braver TS, Barch DM, Cohen JD. Cognition and control in schizophrenia: a computational model of dopamine and prefrontal function. Biol Psychiatry 1999;46:31228. 
Figure 1: Visualization of Marr's three levels of analyses. These distinct levels (computational, algorithmic, implementational) were originally introduced as an approach for relating information processing levels to neuroscience levels to advance the understanding the cognitive and neural mechanisms of vision. Interestingly, this distinction has since been used in research across other cognitive domains (e.g., memory, attention, learning), though many have not strictly adopted the classically rigid framework of Marr's hierarchy. Instead the hierarchy is most commonly used as an organizing principle to highlight distinct conceptual questions at different analysis levels. The current chapter aims to recast Marr's analysis levels and apply them to recent computational models of cognitive control, in order to distill prominent aspects of control mechanisms and highlight different theories about how these mechanisms facilitate temporally extended goal-directed behavior. Thus, we propose that Marr's levels serve a useful purpose in highlighting complementary questions and perspectives that can enhance model-building efforts performed across these three levels.

1

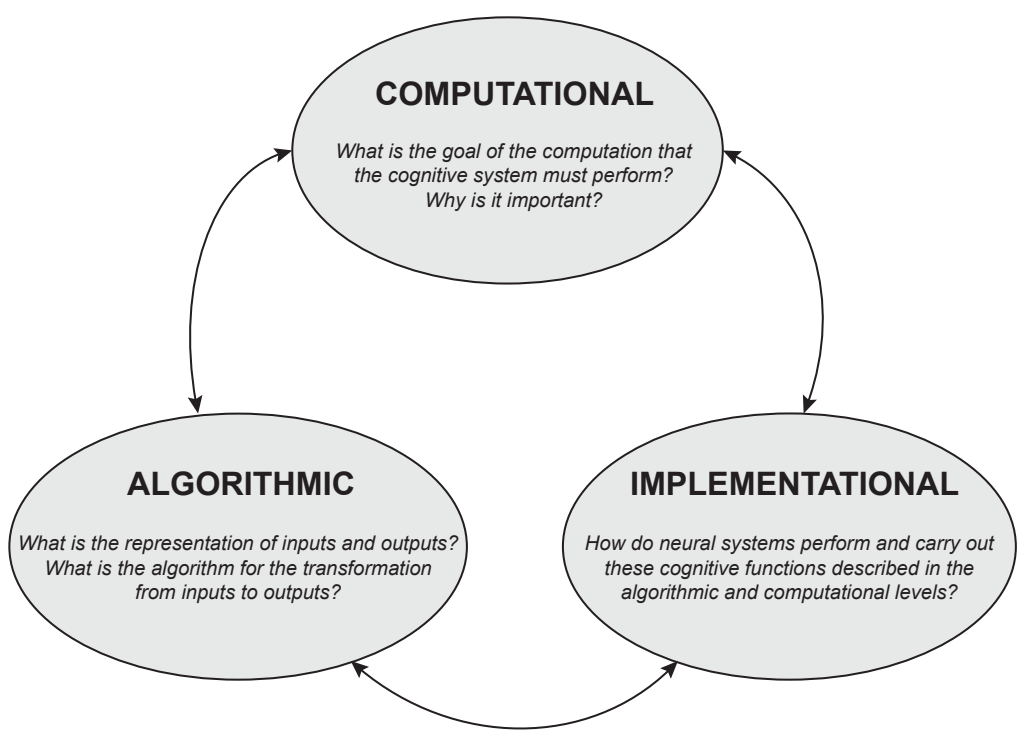


Figure 2: A) Gating Mechanism from Frank and O'Reilly's Prefrontal Basal Ganglia and Working Memory (PBWM) model (Frank, Loughry, and O'Reilly 2001). At the algorithmic level, this connectionist computational model features a gating function, which switches between active maintenance and flexible updating of working memory to incorporate task-relevant information, two core functions of cognitive control. B) Neural Network Model Implementation of the $\boldsymbol{P B W M}$. Here, sensory inputs are mapped onto motor outputs via posterior ("hidden") layers. The prefrontal cortex (PFC) contextualizes this information and maps relevant prior information and goals. The basal ganglia (BG) updates the PFC via dynamic gating, which is driven by dopaminergic (DA) modulation from a separate PVLV system (O'Reilly et al. 2007). Specifically, DA is excitatory onto the Go neurons via D1 receptors and inhibitory onto NoGo neurons via D2 receptors. Thus, increased DA firing will inhibit $\mathrm{SNr}$ (substantia nigra pars reticulata) and disinhibit PFC to facilitate flexible updating of working memory representations in PFC, whereas decreased DA firing counteracts this effect and facilitates active maintenance of current working memory representations in PFC.

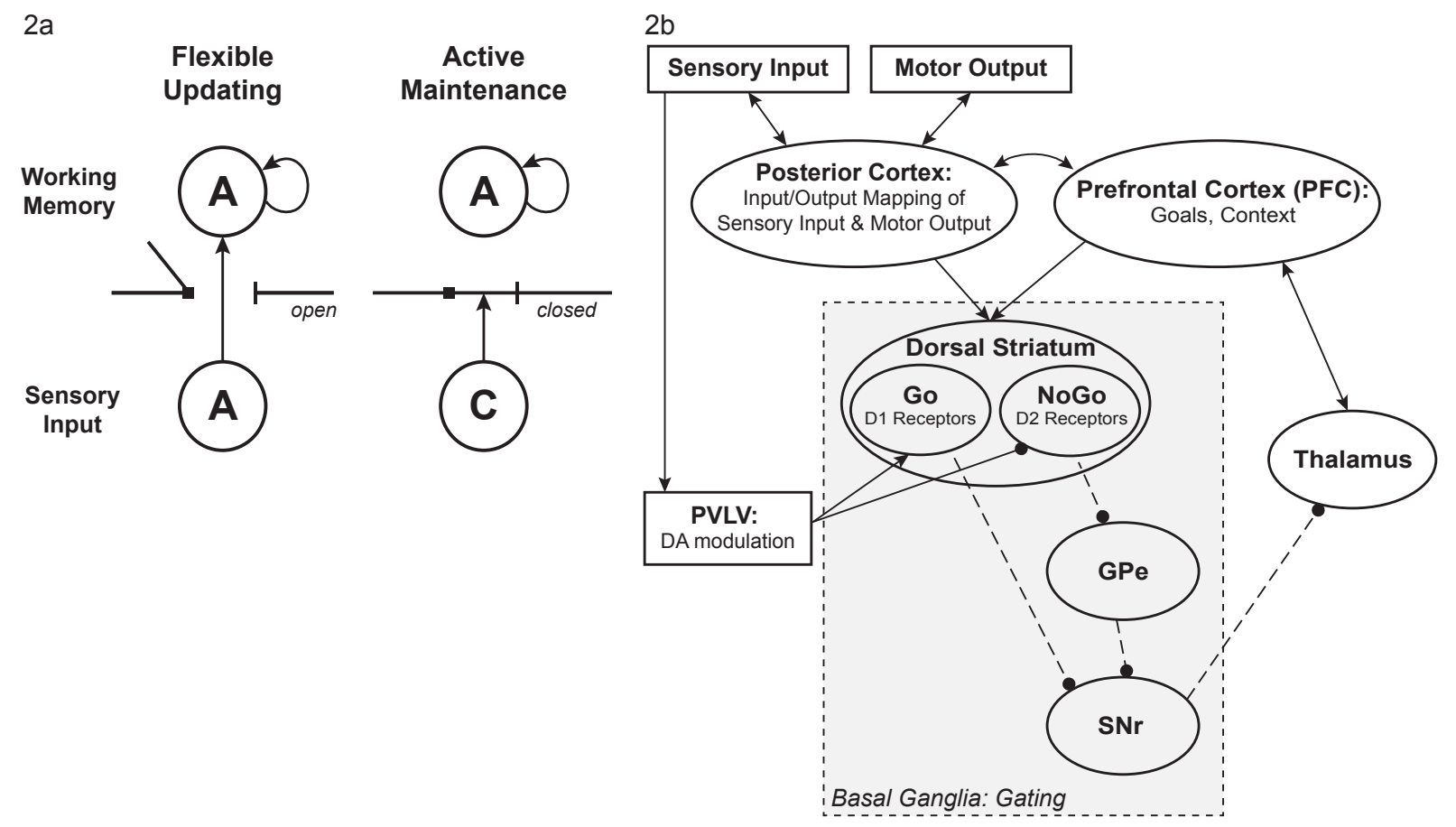


Figure 3: Schematic of the Prediction-Response Outcome (PRO) model by Alexander and Brown $(2011,2014)$. First, the PRO model learns predictions of multiple possible future outcomes of various chosen actions (indicated by $\mathrm{V}_{\mathrm{i}, \mathrm{t}}$ ), using an error likelihood signal. Thus, activity in the PRO model reflects a temporally discounted prediction of such outcomes, which are proportionate to their likelihood of occurrence. Second, the PRO detects discrepancies between predicted and observed outcomes, and uses their prediction error signal $(\delta)$ to update and improve subsequent predictions. In the model below, S refers to the representation of the stimulus (e.g., conflicting arrows from the Erikson flanker task) or task-related feedback (e.g., a screen indicating an error was made). Thus, the PRO model continually learns and updates associations between task-related cues and feedback in cognitive tasks.

3

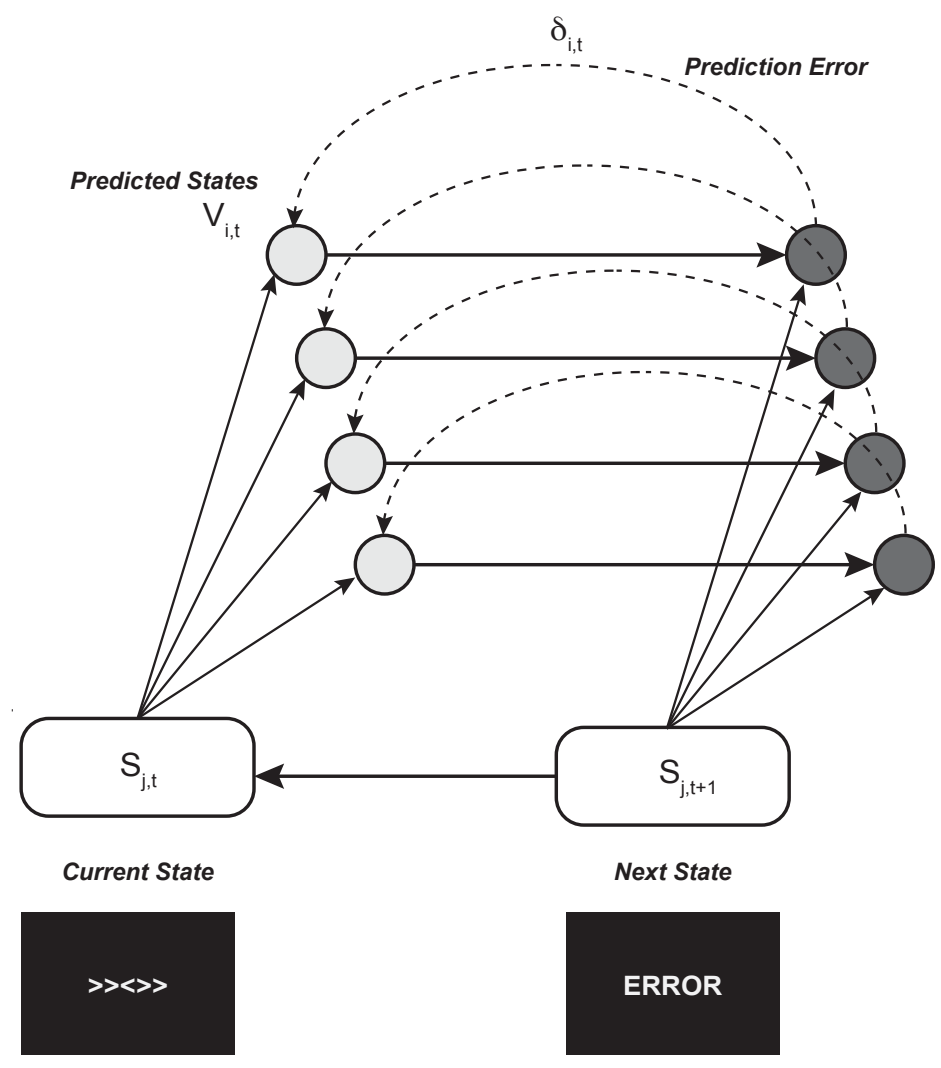


Figure 4: Model of Hierarchical Cognitive Control by Koechlin and colleagues (2003, 2007). This information-theoretic model posits that cognitive control operates according to three nested levels of control processes (branching, episodic, contextual), which are implemented as a cascade from anterior to posterior prefrontal regions. H(a) represents the total amount of information required to select action a, and is a sum of two control terms: bottom-up information conveyed by the stimulus $[\mathrm{I}(\mathrm{s}, \mathrm{a})]$ and top-down information processed in the posterior lateral PFC [Q(a|s)]. This contextual control term Q(a|s) is also nested, and comprised of bottom up information fom the contextual signals and stimulus $[\mathrm{I}(\mathrm{c}, \mathrm{a} \mid \mathrm{s})]$ and top-down information processed in anterior lateral PFC $[\mathrm{Q}(\mathrm{a} \mid \mathrm{s}, \mathrm{c})]$. This latter term represents episodic control, which is the sum of bottom-up information from past event $\mathrm{u}[\mathrm{I}(\mathrm{u}, \mathrm{a} \mid \mathrm{s}, \mathrm{c})]$ and top-down information processed in the polar lateral PFC $[\mathrm{Q}(\mathrm{a} \mid \mathrm{s}, \mathrm{c}, \mathrm{u})]$. The branching control term $[\mathrm{Q}(\mathrm{a} \mid \mathrm{s}, \mathrm{c}, \mathrm{u})]$ relates to information conveyed by events prior to event $\mathrm{u}$, and are maintained until the current episode or trial is complete. Thus, this computational model parses different levels of control based on how much information must be internally represented and actively maintained in order to select and perform a correct action.

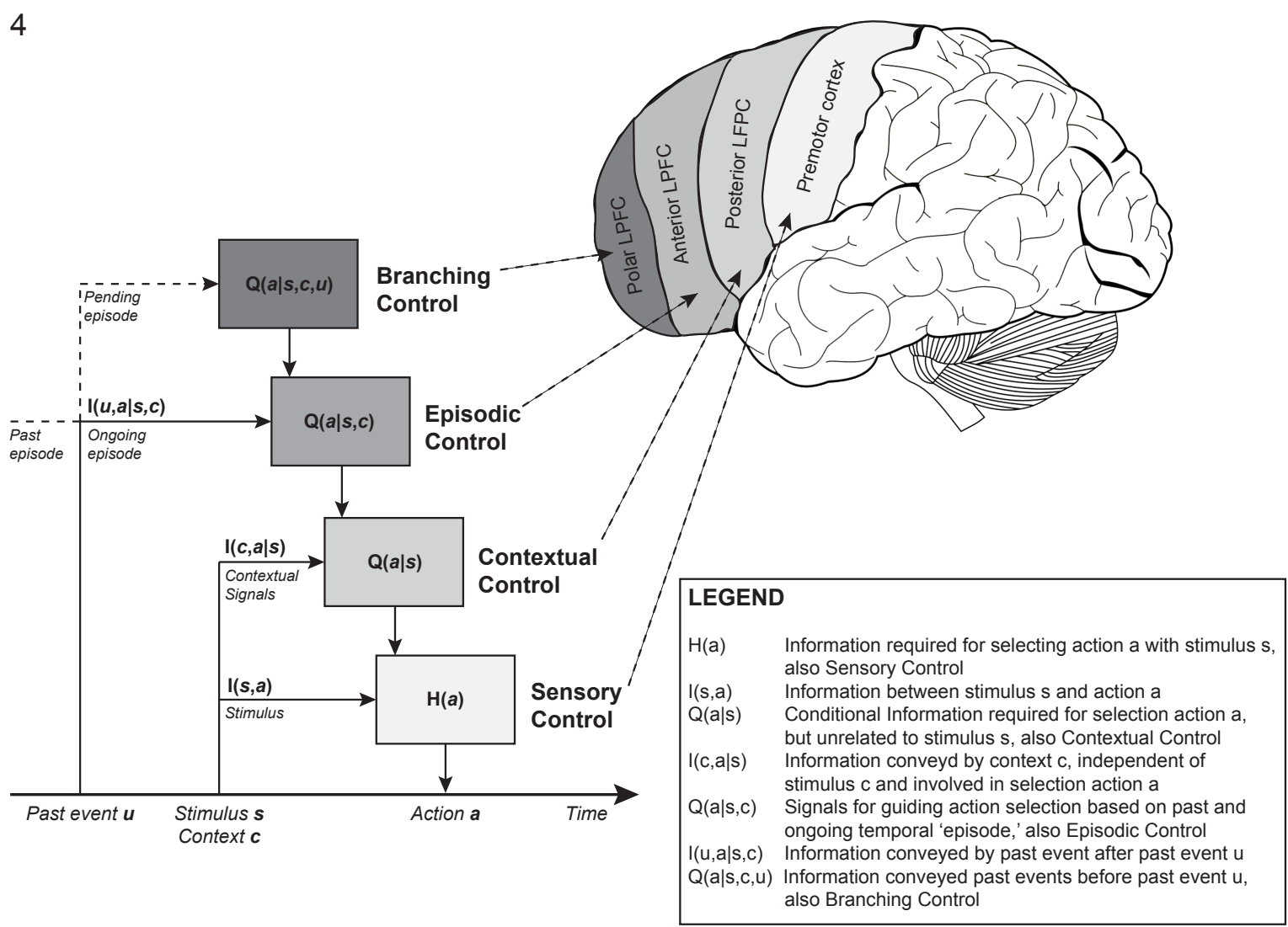


Figure 5: A) Context-Task Set (C-TS) Model by Collins and Frank (2013). This model solves the problem of how to learn hidden task-set rules (i.e., when in a given state and presented with sensory input, which action should be taken in order to maximize reward). The C-TS model posits that states are determined hierarchically; that is, an agent will consider some input dimension to act as a higher order context (C), which indicates a task-set (TS) and other dimensions to as lower level stimuli (S) to determine which motor actions (A) to produce. Here, the color context determines a latent task-set that facilitates learn of shape stimulus-action associations in the learning phase (e.g., $\mathrm{C} 1$ is associated with TS1). In the test phase, C3 maps onto the same shape stimulus-action association as C1, so the C3 transfer context linked to TS1, whereas $\mathrm{C} 4$ should be assigned to a new task set. Critically, the model predicts that it should be faster to transfer a task-set than learning a new task-set. B) Schematic of two-loop corticostriatal gating neural network model. These two loops are nested hierarchically, such that one loop learns to gate an abstract task-set (and cluster contexts associated the same task-sets), whereas the other loop learns to gate a motor action response conditioned on the task-set and perceptual stimulus. Here, color context (C) serves as the input for learning to select the correct task-set (TS) in the prefrontal cortex loop. This information is multiplex with the shape stimulus in parietal cortex to modulate the motor loop and select the correct motor actions. Critically, these two loops accomplish two objectives: 1) constrain motor actions until a task-set is selected, and 2) allow conflict at the level of task-set selection to delay responding in the motor loop, preventing premature action selection until a valid task-set is selected. Taken together, both algorithmic and neural network models similarly and accurately predict behavioral task performance. The synergism of different modeling levels provides an account of how humans engage cognitive control and learning to produce structured abstract representations that enable generalization in the long-term, even if it may be costly in the short-term.

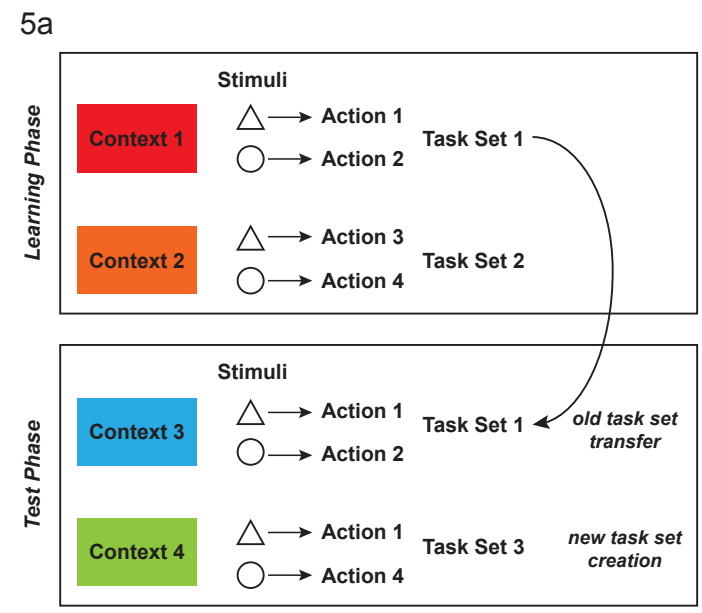

$5 b$

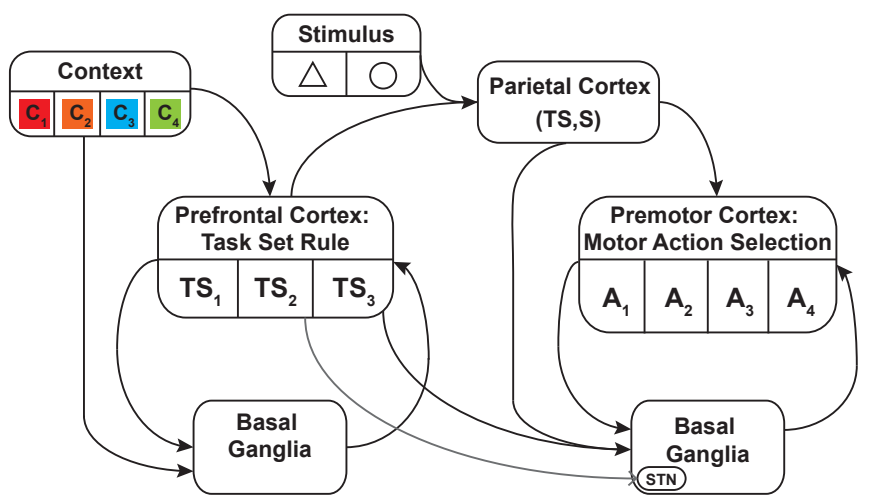




\section{REFERENCES}

Alexander, William H., and Joshua W. Brown. 2011. "Medial Prefrontal Cortex as an ActionOutcome Predictor.” Nature Neuroscience 14 (10). Nature Publishing Group: 1338-44. doi:10.1038/nn.2921.

- 2014. "A General Role for Medial Prefrontal Cortex in Event Prediction." Frontiers in Computational Neuroscience 8 (July): 1-11. doi:10.3389/fncom.2014.00069.

_. 2015. "Hierarchical Error Representation: A Computational Model of Anterior Cingulate and Dorsolateral Prefrontal Cortex." Neural Computation 27: 2354-2410.

Anderson, John R. 1996. "A Simple Theory of Complex Cognition." American Psychologist 51 (4): 355-65. doi:10.1037//0003-066X.51.4.355.

Anderson, John R., Jon M. Fincham, Yulin Qin, and Andrea Stocco. 2008. "A Central Circuit of the Mind." Trends in Cognitive Sciences 12 (4): 136-43. doi:10.1016/j.tics.2008.01.006.

Baddeley, Alan D. 1986. Working Memory. Oxford: Oxford University Press.

Badre, David. 2008. "Cognitive Control, Hierarchy, and the Rostro-Caudal Organization of the Frontal Lobes." Trends in Cognitive Sciences 12 (5): 193-200. doi:10.1016/j.tics.2008.02.004.

Badre, David, and Mark D’Esposito. 2007. "Functional Magnetic Resonance Imaging Evidence for a Hierarchical Organization of the Prefrontal Cortex." Journal of Cognitive Neuroscience 19: 2082-99. doi:10.1162/jocn.2007.19.12.2082.

- 2009. "Is the Rostro-Caudal Axis of the Frontal Lobe Hierarchical?" Nature Reviews. Neuroscience 10 (9): 659-69. doi:10.1038/nrn2667.

Barch, Deanna M., Cameron S. Carter, Todd S. Braver, Fred W. Sabb, Angus MacDonald, Douglas C. Noll, and Jonathan D. Cohen. 2001. "Selective Deficits in Prefrontal Cortex Function in Medication-Naive Patients With Schizophrenia." Archives of General Psychiatry 58 (3): 280. doi:10.1001/archpsyc.58.3.280.

Barch, Deanna M., and Alan Ceaser. 2012. "Cognition in Schizophrenia: Core Psychological and Neural Mechanisms." Trends in Cognitive Sciences 16 (1): 27-34. doi:10.1016/j.tics.2011.11.015.

Barch, Deanna M., Adam Culbreth, and Julia Sheffield. n.d. "Systems Level Modeling of Cognitive Control in Psychiatric Disorders: A Focus on Schizophrenia." In Computational Psychiatry: Mathematical Modeling of Mental Illness.

Bechtel, William. 1994. "Levels of Description and Explanation in Cognitive Science.” Minds and Machines 4 (1): 1-25. doi:10.1007/BF00974202.

Behrens, Timothy E J, Mark W Woolrich, Mark E Walton, and Matthew F S Rushworth. 2007. "Learning the Value of Information in an Uncertain World." Nature Neuroscience 10 (9): 1214-21. doi:10.1038/nn1954.

Belleville, Sylvie, Howard Chertkow, and Serge Gauthier. 2007. "Working Memory and Control of Attention in Persons with Alzheimer's Disease and Mild Cognitive Impairment." Neuropsychology 21 (4): 458-69. doi:10.1037/0894-4105.21.4.458.

Botvinick, Matthew M. 2008. "Hierarchical Models of Behavior and Prefrontal Function." Trends in Cognitive Sciences 12 (5): 201-8. doi:10.1016/j.tics.2008.02.009.

Botvinick, Matthew M., Todd S. Braver, Deanna M. Barch, Cameron S. Carter, and Jonathan D. Cohen. 2001. "Conflict Monitoring and Cognitive Control." Psychological Review. doi:10.1037/0033-295X.108.3.624.

Botvinick, Matthew M., Yael Niv, and Andrew C. Barto. 2009. "Hierarchically Organized 
Behavior and Its Neural Foundations: A Reinforcement Learning Perspective." Cognition 113 (3). Elsevier B.V.: 262-80. doi:10.1016/j.cognition.2008.08.011.

Botvinick, Matthew M, and Jonathan D Cohen. 2014. "The Computational and Neural Basis of Cognitive Control: Charted Territory and New Frontiers.” Cognitive Science 38 (July): 1249-85. doi:10.1111/cogs.12126.

Botvinick, Matthew M, Jonathan D Cohen, and Cameron S Carter. 2004. "Conflict Monitoring and Anterior Cingulate Cortex: An Update." Trends in Cognitive Sciences 8 (12): 539-46. doi:10.1016/j.tics.2004.10.003.

Braver, Todd S. 2012. "The Variable Nature of Cognitive Control: A Dual Mechanisms Framework." Trends in Cognitive Sciences 16 (2). Elsevier Ltd: 106-13. doi:10.1016/j.tics.2011.12.010.

Braver, Todd S., Deanna M. Barch, and Jonathan D. Cohen. 1999. "Cognition and Control in Schizophrenia: A Computational Model of Dopamine and Prefrontal Function." Biological Psychiatry 46 (3): 312-28. http://www.ncbi.nlm.nih.gov/pubmed/10435197.

Braver, Todd S., and Susan R. Bongiolatti. 2002. "The Role of Frontopolar Cortex in Subgoal Processing during Working Memory." NeuroImage 15 (3): 523-36. doi:10.1006/nimg.2001.1019.

Braver, Todd S., and Jonathan D. Cohen. 1999. "Dopamine, Cognitive Control, and Schizophrenia: The Gating Model.” Progress in Brain Research 121 (2): 327-49. doi:10.1016/S0079-6123(08)63082-4.

- 2000. "On the Control of Control: The Role of Dopamine in Regulating Prefrontal Function and Working Memory." In Attention and Performance XVIII, edited by Stephen Monsell and Jon Driver, 713-37. Cambridge, Massachusetts: MIT Press. doi:10.1016/S0165-0173(03)00143-7.

Braver, Todd S., Jonathan D. Cohen, and Deanna M. Barch. 2002. "The Role of Prefrontal Cortex in Normal and Disordered Cognitive Control: A Cognitive Neuroscience Perspective." In Principles of Frontal Lobe Function, edited by D.T. Stuss and R.T. Knight, 428-48. Oxford, England: Oxford University Press.

Braver, Todd S., Jeremy R. Reynolds, and David I. Donaldson. 2003. "Neural Mechanisms of Transient and Sustained Cognitive Control during Task Switching." Neuron 39 (4): 713-26. doi:10.1016/S0896-6273(03)00466-5.

Braver, Todd S., and Hannes Ruge. 2006. "Functional Neuroimaging of Executive Functions." In Handbook of Functional Neuroimaging of Cognition, edited by R Cabeza and A Kingstone, 2nd ed., 307-48. Cambridge, Massachusetts: MIT Press.

Brown, Joshua W., and Todd S. Braver. 2005. "Learned Predictions of Error Likelihood in the Anterior Cingulate Cortex." Science 307 (5712): 1110-21.

Brown, R.G., and C.D. Marsden. 1990. "Cognitive Function in Parkinson's Disease: From Description to Theory." Trends in Neurosciences 13 (1): 21-29. doi:10.1016/01662236(90)90058-I.

Carter, Cameron S., Todd S. Braver, Deanna M. Barch, Matthew M. Botvinick, Douglas Noll, and Jonathan D. Cohen. 1998. "Anterior Cingulate Cortex, Error Detection, and the Online Monitoring of Performance." Science (New York, N.Y.) 280 (5364): 747-49. doi:10.1126/science.280.5364.747.

Chambon, Valérian, Nicolas Franck, Etienne Koechlin, Eric Fakra, Gabriela Ciuperca, Jean Michel Azorin, and Chlöé Farrer. 2008. "The Architecture of Cognitive Control in Schizophrenia." Brain 131 (4): 962-70. doi:10.1093/brain/awn032. 
Changeux, J P, and S Dehaene. 1989. "Neuronal Models of Cognitive Functions." Cognition 33 (1-2): 63-109. doi:0010-0277(89)90006-1 [pii].

Cohen, Jonathan D., Kevin Dunbar, and James L. McClelland. 1990. "On the Control of Automatic Processes: A Parallel Distributed Processing Account of the Stroop Effect." Psychological Review 97 (3): 332-61. doi:10.1037/0033-295X.97.3.332.

Cohen, Jonathan D., and David Servan-Schreiber. 1992. "Context, Cortex, and Dopamine: A Connectionist Approach to Behavior and Biology in Schizophrenia." Psychological Review 99 (1): 45-77. doi:http://dx.doi.org/10.1037/0033-295X.99.1.45.

Cohen, Jonathan D, Todd S. Braver, and Joshua W. Brown. 2002. "Computational Perspectives on Dopamine Function in Prefrontal Cortex." Current Opinion in Neurobiology, 223-29. http://www.sciencedirect.com/science/article/pii/S0959438802003148.

Cohen, Jonathan D, Todd S Braver, and Randall C O'Reilly. 1996. “A Computational Approach to Prefrontal Cortex , Cognitive Control and Schizophrenia: Recent Developments and Current Challenges." Philosophical Transactions of the Royal Society of London, Series B, Biological Sciences 351: 1515-27.

Collins, Anne G. E. 2017. "The Cost of Structure Learning." Journal of Cognitive Neuroscience 29 (10): 1646-55. doi:10.1162/jocn.

Collins, Anne G. E., and Michael J. Frank. 2013. "Cognitive Control over Learning: Creating, Clustering, and Generalizing Task-Set Structure.” Psychological Review 120 (1): 190-229. doi:10.1037/a0030852.

Compte, Albert, Nicolas Brunel, P S Goldman-Rakic, and X J Wang. 2000. "Synaptic Mechanisms and Network Dynamics Underlying Spatial Working Memory in a Cortical Network Model." Cerebral Cortex (New York, N.Y. : 1991) 10 (9): 910-23. http://www.ncbi.nlm.nih.gov/pubmed/10982751.

Cooper, Richard P., and Tim Shallice. 2006. "Hierarchical Schemas and Goals in the Control of Sequential Behavior.” Psychological Review 113 (4): 887-916. doi:10.1037/0033295X.113.4.887.

D’Ardenne, Kimberlee, Neir Eshel, Joseph Luka, Agatha Lenartowicz, E Leigh, No December, Neir Eshelb, et al. 2012. "Role of Prefrontal Cortex and the Midbrain Dopamine System in Working Memory Updating." Proceedings of the National Academy of Sciences of the United States of America 109 (49): 19900-909. doi:10.1073/pnas.1116727.

Dayan, Peter. 2001. "Levels of Analysis in Neural Modeling." In Encyclopedia of Cognitive Science, 1-10. London, England: MacMillan Press. doi:10.1002/0470018860.s00363.

- 2012. "How to Set the Switches on This Thing." Current Opinion in Neurobiology 22 (6). Elsevier Ltd: 1068-74. doi:10.1016/j.conb.2012.05.011.

Deco, Gustavo, and Edmund T. Rolls. 2003. "Attention and Working Memory: A Dynamical Model of Neuronal Activity in the Prefrontal Cortex." European Journal of Neuroscience 18 (8): 2374-90. doi:10.1046/j.1460-9568.2003.02956.x.

Dixon, Matthew L, and Kalina Christoff. 2012. "The Decision to Engage Cognitive Control Is Driven by Expected Reward-Value: Neural and Behavioral Evidence." PLoS ONE 7 (12). doi:10.1371/journal.pone.0051637.

Duncan, John. 2010. "The Multiple-Demand (MD) System of the Primate Brain: Mental Programs for Intelligent Behaviour.” Trends in Cognitive Sciences 14 (4). Elsevier Ltd: 172-79. doi:10.1016/j.tics.2010.01.004.

Duncan, John, and A M Owen. 2000. "Common Regions of the Human Frontal Lobe Recruted by Diverse Cognitive Demands." Trends in Neurosciences 23 (10): 475-83. 
doi:10.1016/S0166-2236(00)01633-7.

Durstewitz, Daniel, Jeremy K Seamans, and Terrence J Sejnowski. 2000. "Neurocomputational Models of Working Memory.” Nature Neuroscience 3 (11): 1184. doi:10.1038/81460.

Duverne, Sandrine, and Etienne Koechlin. 2017. "Rewards and Cognitive Control in the Human Prefrontal Cortex." Cerebral Cortex, no. September: 1-16. doi:10.1093/cercor/bhx210.

Egner, Tobias. 2009. "Prefrontal Cortex and Cognitive Control: Motivating Functional Hierarchies." Nature Neuroscience 12 (7). Nature Publishing Group: 821-22. doi:10.1038/nn0709-821.

Eliasmith, Chris, Terrence C Stewart, Xuan Choo, Trevor Bekolay, Travis Dewolf, Yichuan Tang, and Daniel Rasmussen. 2012. "A Large-Scale Model of the Functioning Brain." Science 338 (i): 1202-5.

Engle, Randall W., and Michael J. Kane. 2004. "Executive Attention, Working Memory Capacity, and a Two-Factor Theory of Cognitive Control." The Psychology of Learning and Motivation: Advances in Research and Theory, 145-99. doi:10.1016/S00797421(03)44005-X.

Fales, Christina L., Deanna M. Barch, Melissa M. Rundle, Mark A. Mintun, Abraham Z. Snyder, Jonathan D. Cohen, Jose Mathews, and Yvette I. Sheline. 2008. "Altered Emotional Interference Processing in Affective and Cognitive-Control Brain Circuitry in Major Depression.” Biological Psychiatry 63 (4): 377-84. doi:10.1016/j.biopsych.2007.06.012.

Frank, Michael J, and David Badre. 2012. "Mechanisms of Hierarchical Reinforcement Learning in Corticostriatal Circuits 1: Computational Analysis." Cerebral Cortex (New York, N.Y. : 1991) 22 (3): 509-26. doi:10.1093/cercor/bhr114.

Frank, Michael J, Bryan Loughry, and Randall C. O'Reilly. 2001. "Interactions between Frontal Cortex and Basal Ganglia in Working Memory: A Computational Model." Cognitive, Affective \& Behavioral Neuroscience 1 (2): 137-60. http://www.ncbi.nlm.nih.gov/pubmed/12467110.

Fuster, Joaquim M. 2001. "The Prefrontal Cortex--an Update. Time Is of the Essence." Neuron 30: 319-33.

Gehring, William J, Brian Goss, Michael G H Coles, David E Meyer, and Emanuel Donchin. 1993. "A Neural System for Error Detection and Compenstation." Psychological Science 4 (6): 385-90. doi:10.1111/j.1467-9280.1993.tb00586.x.

Gershman, Samuel J., Jonathan D. Cohen, and Yael Niv. 2010. "Learning to Selectively Attend." 32nd Annual Proceedings of the Cognitive Science Society, 1270-75. http://csjarchive.cogsci.rpi.edu/proceedings/2010/papers/0359/paper0359.pdf.

Greisberg, Scott, and D McKay. 2003. "Neuropsychology of Obsessive-Compulsive Disorder: A Review and Treatment Implications." Clin Psychol Rev 23 (1): 95-9117. doi:10.1016/S0272-7358(02)00232-5.

Halari, Rozmin, Mima Simic, Carmine M. Pariante, Andrew Papadopoulos, Anthony Cleare, Michael Brammer, Eric Fombonne, and Katya Rubia. 2009. "Reduced Activation in Lateral Prefrontal Cortex and Anterior Cingulate during Attention and Cognitive Control Functions in Medication-Naïve Adolescents with Depression Compared to Controls." Journal of Child Psychology and Psychiatry and Allied Disciplines 50 (3): 307-16. doi:10.1111/j.14697610.2008.01972.x.

Hazy, T. E., M. J. Frank, and R. C. O’Reilly. 2007. “Towards an Executive without a Homunculus: Computational Models of the Prefrontal Cortex/basal Ganglia System." Philosophical Transactions of the Royal Society B: Biological Sciences 362 (1485): 1601- 
13. doi:10.1098/rstb.2007.2055.

Hinton, Geoffrey E. 1984. "Distributed Representations." Technical Report CMU-CS-84-157.

Holroyd, Clay B., and Samuel M. McClure. 2015. "Hierarchical Control over Effortful Behavior by Rodent Medial Frontal Cortex: A Computational Model." Psychological Review 122 (1): 54-83. doi:10.1037/a0038339.

Holroyd, Clay B., and N Yeung. 2011. "An Integrative Theory of Anterior Cingulate Cortex Function: Option Selection in Hierarchical Reinforcement Learning." In The Neural Basis of Motivational and Cognitive Control, 333-49. Cambridge, Massachusetts: MIT Press. http://books.google.com/books?hl=en\&lr=\&id=A_eoYgtLmFMC\&oi=fnd\&pg=PA333\&dq $=$ An + integrative + theory + of + anterior + cingulate + cortex + function: + Option + selection + in + hie rarchical+reinforcement+learning\&ots $=$ YcpRBOliOW\&sig=6rPHmu8F6dIjIdrW5SOBmJcOE8.

Holroyd, Clay B., Nick Yeung, Michael G. H. Coles, and Jonathan D. Cohen. 2005. "A Mechanism for Error Detection in Speeded Response Time Tasks." Journal of Experimental Psychology: General 134 (2): 163-91. doi:10.1037/0096-3445.134.2.163.

Holroyd, Clay B, and M. G. H. Coles. 2002. "The Neural Basis of Human Error Processing: Reinforcement Learning, Dopamine, and the Error-Related Negativity.” Psychological Review 109 (4): 679-709. doi:10.1037//0033-295X.109.4.679.

Holroyd, Clay B, Sander Nieuwenhuis, Nick Yeung, Leigh Nystrom, Rogier B Mars, Michael G H Coles, and Jonathan D Cohen. 2004. "Dorsal Anterior Cingulate Cortex Shows fMRI Response to Internal and External Error Signals." Nature Neuroscience 7 (5): 497-98. doi: $10.1038 / \mathrm{nn} 1238$.

Kerns, J. G. 2004. "Anterior Cingulate Conflict Monitoring and Adjustments in Control." Science 303 (5660): 1023-26. doi:10.1126/science.1089910.

Kieras, David E, and David E Meyer. 1997. "An Overview of the EPIC Architecture for Cognition and Performance With Application to Human-Computer Interaction." HumanComputer Interaction 12: 391-438.

Koechlin, Etienne, Chrystèle Ody, and Frédérique Kouneiher. 2003. "The Architecture of Cognitive Control in the Human Prefrontal Cortex." Science 302 (November): 1181-86.

Koechlin, Etienne, and Christopher Summerfield. 2007. "An Information Theoretical Approach to Prefrontal Executive Function." Trends in Cognitive Sciences 11 (6): 229-35. doi:10.1016/j.tics.2007.04.005.

Lesh, Tyler A, Tara A Niendam, Michael J Minzenberg, and Cameron S Carter. 2011. "Cognitive Control Deficits in Schizophrenia: Mechanisms and Meaning." Neuropsychopharmacology 36. Nature Publishing Group: 316-38. doi:10.1038/npp.2010.156.

Maia, Tiago V., and Michael J. Frank. 2017. "An Integrative Perspective on the Role of Dopamine in Schizophrenia." Biological Psychiatry 81 (1). Elsevier: 52-66. doi:10.1016/j.biopsych.2016.05.021.

Marr, David. 1982. "Chapter 1: The Philosophy and the Approach.” In Vision. San Francisco: W.H. Freeman and Co.

Meltzer, H Y, and S M Stahl. 1976. "The Dopamine Hypothesis of Schizophrenia: A Review." Schizophrenia Bulletin 2 (1): 19-76.

Nee, Derek Evan, and Joshua W. Brown. 2013. "Dissociable Frontal-Striatal and Frontal-Parietal Networks Involved in Updating Hierarchical Contexts in Working Memory." Cerebral Cortex 23 (9): 2146-58. doi:10.1093/cercor/bhs 194. 
Nee, Derek Evan, and Mark D'Esposito. 2016. "The Hierarchical Organization of the Lateral Prefrontal Cortex.” eLife 5 (MARCH2016): 1-26. doi:10.7554/eLife.12112.

Newell, Allen, and HA Simon. 1972. Human Problem Solving. http://www.sci.brooklyn.cuny.edu/ kopec/cis718/fall_2005/2/Rafique_2_humanthinking.do c.

Newell, Allen, and Herbert A. Simon. 1961. "Computer Simulation of Human Thinking." Science 134 (3495): 2011-17. doi:10.2307/1708146.

Norman, Donald A, and Tim Shallice. 1986. "Attention to Action: Willed and Automatic Control of Behavior." In Conciousness and Self-Regulation: Advances in Research and Theory, edited by R Davidson, G Schwartz, and D Shapiro, 1-18. New York.

O’Reilly, Randall C. 2006. "Biologically Based Computational Models of High-Level Cognition.” Science 314 (October): 91-94. doi:10.1126/science.1127242.

O'Reilly, Randall C., Seth A. Herd, and Wolfgang M. Pauli. 2010. "Computational Models of Cognitive Control.” Current Opinion in Neurobiology 20 (2). Elsevier Ltd: 367-77. doi:10.1016/j.conb.2010.01.008.

O’Reilly, Randall C, Todd S Braver, and Jonathan D Cohen. 1999. “A Biologically-Based Computational Model of Working Memory." In Models of Working Memory: Mechanisms of Active Maintenance and Executive Control, 375-411. doi:10.1017/CBO9781139174909.

O’Reilly, Randall C, and Michael J Frank. 2006. "Making Working Memory Work: A Computational Model of Learning in the Prefrontal Cortex and Basal Ganglia." Neural Computation 18 (2): 283-328. doi:10.1162/089976606775093909.

O'Reilly, Randall C, Michael J Frank, Thomas E Hazy, and Brandon Watz. 2007. "PVLV: The Primary Value and Learned Value Pavlovian Learning Algorithm.” Behavioral Neuroscience 121 (1): 31-49. doi:10.1037/0735-7044.121.1.31.

Petrides, M. 2005. "Lateral Prefrontal Cortex: Architectonic and Functional Organization." Philosophical Transactions of the Royal Society B: Biological Sciences 360 (1456): 781-95. doi:10.1098/rstb.2005.1631.

Poldrack, Russell a. 2007. "Region of Interest Analysis for fMRI." Social Cognitive and Affective Neuroscience 2 (1): 67-70. doi:10.1093/scan/nsm006.

Polyn, Sean M., Kenneth A. Norman, and Michael J. Kahana. 2009. "A Context Maintenance and Retrieval Model of Organizational Processes in Free Recall." Psychological Review 116 (1): 129-56. doi:10.1037/a0014420.

Posner, Michael I, and Charles R.R. Snyder. 1975. “Attention and Cognitive Control.” In Information Processing and Cognition: The Loyola Symposium, edited by R.L. Solso, 5585. Hillsdale, New Jersey: Lawrence Erlbaum Assosciates, Inc.

Reynolds, Jeremy R., and Randall C. O'Reilly. 2009. "Developing PFC Representations Using Reinforcement Learning.” Cognition 113 (3). Elsevier B.V.: 281-92. doi:10.1016/j.cognition.2009.05.015.

Reynolds, Jeremy R., Randall C. O'Reilly, Jonathan D. Cohen, and Todd S. Braver. 2012. "The Function and Organization of Lateral Prefrontal Cortex: A Test of Competing Hypotheses." PLoS ONE 7 (2). doi:10.1371/journal.pone.0030284.

Ridderinkhof, K. Richard, Markus Ullsperger, Eveline A. Crone, and Sander Nieuwenhuis. 2004. "The Role of the Medial Frontal Cortex in Cognitive Control." Science 306: 443-47.

Rogers, Timothy T., and James L. McClelland. 2004. Semanitc Cognition: A Parallel Distributed Processing Approach. MIT Press.

Rolls, Edmund T., Marco Loh, Gustavo Deco, and Georg Winterer. 2008. “Computational 
Models of Schizophrenia and Dopamine Modulation in the Prefrontal Cortex." Nature Reviews Neuroscience 9 (9): 696-709. doi:10.1038/nrn2462.

Rolls, Edmund T, and Fabian Grabenhorst. 2008. "The Orbitofrontal Cortex and beyond: From Affect to Decision-Making." Progress in Neurobiology 86 (3): 216-44. doi:10.1016/j.pneurobio.2008.09.001.

Rumelhart, David E, James L McClelland, and PDP Research Group. 1987. Parallel Distributed Processing, Vol 1. Cambridge, Massachusetts: MIT Press.

Rumelhart, David E, P. Smolensky, J. L. McClelland, and G. E. Hinton. 1986. "Schemata and Sequential Thought Processes in PDP Models." In Parallel Distributed Processing, Vol. 2: Explorations, 7-57. doi:10.1016/B978-1-4832-1446-7.50020-0.

Schneider, Walter, and Jason M. Chein. 2003. "Controlled \& Automatic Processing: Behavior, Theory, and Biological Mechanisms." Cognitive Science 27 (3): 525-59. doi:10.1016/S0364-0213(03)00011-9.

Seeman, Philip. 1987. "Dopamine-Receptors and the Dopamine Hypothesis of Schizophrenia." Synapse 1 (2): 133-52. isi:A1987K946100002.

Shannon, Claude E. 1948. "A Mathematical Theory of Communication." The Bell System Technical Journal 27 (July 1928): 379-423. doi:10.1145/584091.584093.

Shenhav, Amitai, Matthew M Botvinick, and Jonathan D Cohen. 2013. "The Expected Value of Control: An Integrative Theory of Anterior Cingulate Cortex Function." Neuron 79 (2). Elsevier Inc.: 217-40. doi:10.1016/j.neuron.2013.07.007.

Shenhav, Amitai, Sebastian Musslick, Falk Lieder, Wouter Kool, Thomas L. Griffiths, Jonathan D. Cohen, and Matthew M. Botvinick. 2017. "Toward a Rational and Mechanistic Account of Mental Effort." Annual Review of Neuroscience 40 (1): 99-124. doi:10.1146/annurevneuro-072116-031526.

Shiffrin, Richard M., and Walter Schneider. 1977. "Controlled and Automatic Human Information Processing: II. Perceptual Learning, Automatic Attending and a General Theory." Psychological Review 84 (2): 127-90. doi:10.1037/0033-295X.84.2.127.

Shima, Keisetsu, Masaki Isoda, Hajime Mushiake, and Jun Tanji. 2007. "Categorization of Behavioural Sequences in the Prefrontal Cortex." Nature 445 (7125): 315-18. doi:10.1038/nature05470.

Simon, Herbert A. 1962. "The Architecture of Complexity." Proceedings of the American Philosophical Society 106 (6): 467-82. doi:10.1007/978-3-642-27922-5_23.

Snitz, Beth E., Angus W. MacDonald, and Cameron S. Carter. 2006. "Cognitive Deficits in Unaffected First-Degree Relatives of Schizophrenia Patients: A Meta-Analytic Review of Putative Endophenotypes." Schizophrenia Bulletin 32 (1): 179-94. doi:10.1093/schbul/sbi048.

Snyder, Hannah R., Akira Miyake, and Benjamin L. Hankin. 2015. “Advancing Understanding of Executive Function Impairments and Psychopathology: Bridging the Gap between Clinical and Cognitive Approaches." Frontiers in Psychology 6 (MAR). doi:10.3389/fpsyg.2015.00328.

Snyder, Solomon H. 1976. "The Dopamine Hypothesis of Schizophrenia: Focus on the Dopamine Receptor.” American Journal of Psychiatry 133 (2): 197-202. doi:10.1176/ajp.133.2.197.

Solway, Alec, Carlos Diuk, Natalia Cordova, Debbie Yee, Andrew G. Barto, Yael Niv, and Matthew M. Botvinick. 2014. "Optimal Behavioral Hierarchy." PLoS Computational Biology 10 (8): e1003779. doi:10.1371/journal.pcbi.1003779. 
Tenenbaum, Joshua B., Thomas L. Griffiths, and Charles Kemp. 2006. "Theory-Based Bayesian Models of Inductive Learning and Reasoning." Trends in Cognitive Sciences 10 (7): 309_ 18. doi:10.1016/j.tics.2006.05.009.

Toda, Mitsuru, and Anissa Abi-Dargham. 2007. "Dopamine Hypothesis of Schizophrenia: Making Sense of It All." Current Psychiatry Reports 9 (4): 329-36. doi:10.1007/s11920007-0041-7.

Vaidya, C.J., S.A. Bunge, N.M. Dudukovic, C.A. Zalecki, G.R. Elliott, and J.D. Gabrieli. 2005. "Altered Neural Substrates of Cognitive Control in Childhood ADHD: Evidence from Functional Magnetic Resonance Imaging.” Am.J.Psychiatry 162 (9): 1605-13.

van Meel, Catharina S., Dirk J. Heslenfeld, Jaap Oosterlaan, and Joseph A. Sergeant. 2007. "Adaptive Control Deficits in Attention-Deficit/hyperactivity Disorder (ADHD): The Role of Error Processing." Psychiatry Research 151 (3): 211-20. doi:10.1016/j.psychres.2006.05.011.

Vassena, Eliana, Clay B. Holroyd, and William H. Alexander. 2017. "Computational Models of Anterior Cingulate Cortex: At the Crossroads between Prediction and Effort." Frontiers in Neuroscience 11 (JUN): 1-9. doi:10.3389/fnins.2017.00316.

Velanova, Katerina, Larry L Jacoby, Mark E Wheeler, Mark P McAvoy, Steve E Petersen, and Randy L Buckner. 2003. "Functional-Anatomic Correlates of Sustained and Transient Processing Components Engaged during Controlled Retrieval." The Journal of Neuroscience 23 (24): 8460-70. doi:23/24/8460 [pii].

Wang, Xiao-Jing. 2013. “The Prefrontal Cortex as a Quintessential 'Cognitive-Type' Neural Circuit." In Principles of Frontal Lobe Function, edited by Donald T Stuss and Robert T. Knight, 2nd ed., 226-48. New York: Oxford University Press.

Westbrook, Andrew, and Todd S. Braver. 2016. "Dopamine Does Double Duty in Motivating Cognitive Effort." Neuron 89 (4). Elsevier Inc.: 695-710. doi:10.1016/j.neuron.2015.12.029.

Wylie, Scott A, K Richard Ridderinkhof, Theodore R Bashore, and Wery P M van den Wildenberg. 2010. "The Effect of Parkinson'S Disease on the Dynamics of On-Line and Proactive Cognitive Control during Action Selection." Journal of Cognitive Neuroscience 22 (9): 2058-73.

Zipser, D, B Kehoe, G Littlewort, and J Fuster. 1993. "A Spiking Network Model of Short-Term Active Memory.” The Journal of Neuroscience 13 (8): 3406-20. doi:10.1162/jocn_a_00061. 